\title{
Analyzing Brainwaves While Listening To Quranic Recitation Compared With Listening To Music Based on EEG Signals
}

\author{
Sabaa Ahmed Yahya Al-Galal, Imad Fakhri Taha Alshaikhli \\ Dept of Computer Science, International Islamic University Malaysia, Kuala Lumpur, Malaysia. \\ aljalalsaba@gmail.com \\ Dept of Computer Science, International Islamic University Malaysia, Kuala Lumpur, Malaysia. \\ imadf@iium.edu.my
}

\begin{abstract}
The electroencephalogram (EEG) is an acquiring of the electrical fluctuations that occur in the brain, generated simply by positioning electrodes over the scalp with amplification of the electrical potential produced. The actual EEG indicates three primary types of wave, named alpha, beta and delta, which are distinct based on their rate of production. Data has been collected from twenty five subjects using BrainMarker EEG hardware and software. Brainwaves were measured, focusing on the Alpha and Beta bands to measure subjects' calmness while listening to Quran recitation compared with relaxing music. The result showed that higher alpha magnitudes were generated during listening to Quran recitation.
\end{abstract}

Keywords- EEG , Brainwaves, Alpha, Beta, Quran recitation, Relaxing music.

\begin{abstract}
I. INTRODUCTION
Brainwaves is becoming an important induction of the peoples' stress level, Electrophysiology is the area associated with physiology that is concerned with the electrical phenomena related to the nervous system as well as other physiological activities. Recently numerous studies shed the light on investigating the brainwaves through recorded EEG. The outcomes of such studies can be very useful in many aspects, particularly emotional and physiological health issues [1]-[3]. In this research, we aim to investigate how Quran recitation as sound therapy can reduce levels of stress and anxiety for subjects compared with relaxation music by analysing acquired EEG signals.
\end{abstract}

\section{Related Studies}

Electroencephalograms signals are categorized into five types of waves (delta, theta, alpha, beta and gamma) [4]. According to the aforementioned reference, alpha wave frequencies lie between $8-13 \mathrm{~Hz}$ and are associated with relaxed awareness. Most subjects produce alpha waves while relaxed with their eyes closed. Beta waves (14-26 Hz) are associated with active thinking and external focus. Waves greater than $26 \mathrm{~Hz}$ are called Gamma waves or sometimes fast Beta waves; the amplitudes of these rhythms are very low, and their occurrence is rarely observed. Detection of these rhythms can be used to confirm certain brain diseases.

One major theory of emotions proposes that EEG can be used to categorize a basic set of human emotions [5]. Each emotion is distinct from other emotions as perceived by its psychological and physiological manifestations.

Typically the Circumplex model of emotion originated by James Russell [6], This specific model implies that emotions can be scattered within a two-dimensional a circular space, comprised of arousal and valence dimensions. Arousal symbolizes the vertical axis and also valence symbolizes the horizontal axis, as you move the core circle presents a neutral valence and also a medium level of arousal. Within this model, emotional states can certainly be indicated at virtually any level of valence and arousal, or perhaps within a neutral level of either of such elements. Circumplex models have been employed mostly to evaluate stimulus associated with emotion terms, cosmetic expression, and also affective states. As illustrated in the figure 1.

Russell explained the built circumplex model as representative of core affect, or perhaps the most basic emotions which are not essentially targeted at something. Various prototypical psychological episodes, as well as obvious 
feelings that happen to be evoked or simply redirected through particular objects can certainly be plotted over the circumplex, based on the levels of arousal and valence [6].

According to [4], EEG signals show increased alpha waves and reduced beta waves while listening to Quranic recitation. In contrast, beta waves increase when listening to rock music. Another study compared the alpha waves of subjects listening to Quranic recitation and classical music. The results showed that $12.67 \%$ of the samples demonstrated increases in the alpha band during Quranic recitation, while $9.97 \%$ of samples showed similar increases for classical music [7]. To the best of our knowledge, there is no existing methodological study that uses ECG to investigate the heart's electrical activity while listening to Quranic recitation.

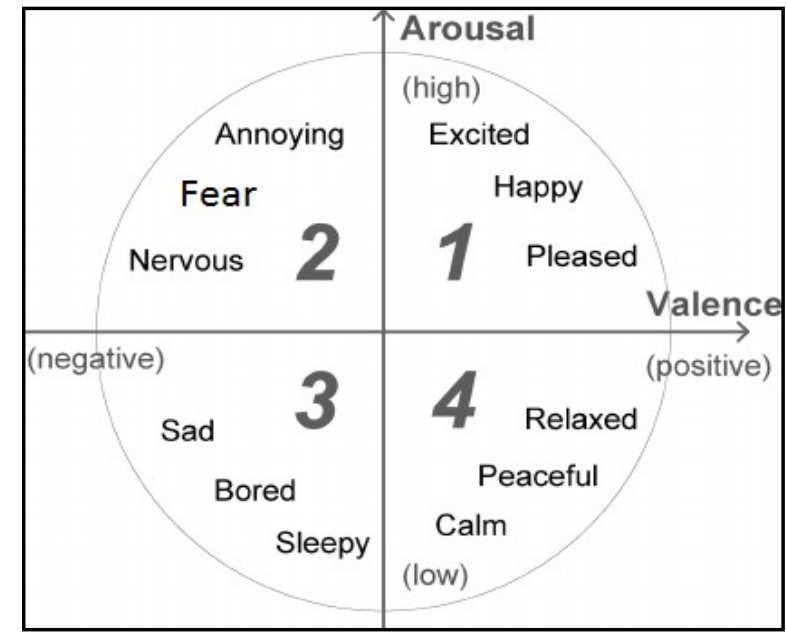

Fig. 1 Circumplex model of affect [6]

\section{Methodology}

The methodology of this study is divided into two mean directions; first is the experiment and data collection, and next is the data analysis.

\section{A. Experiment setup}

Following are the main steps in our experiment:

1) Material: In the present study, we employed IAPS [8] combined with suitable music for each distinct emotion because it has been asserted that combinations of pictures and sounds give better effect [9]. For the test stimulus, there are two sets of excerpts: Quran recitation and relaxing music. We chose three Quranic excerpts Surat Yasin and Al-Inshirah, and AlMu'awwidhatayn with Al-Ikhlas lasted one minute each. For music track selection to the best of our knowledge the following are amongst the best known types of relaxing music: Mozart Music K448, New age and Jazz [10]-[12]. The music tracks had been chosen very carefully according to music websites built using emotion as a cue for music tracks, such as Musicovery, AMG and Last.fm. The three tracks were chosen from the calm quadrant.

2) Electrode placements: EEG was acquired by placing nineteen surface electrodes on the scalp according to the international 10-20 system (Fp1, Fp2, F7, F8, F3, F4, Fz, T7, T8, C3, C4, Cz, P7, P8, P3, P4, Pz, O1 and O2) We used BrainMarker software and Hardware as shown in figure 2. The sampling rate was set to $250 \mathrm{~Hz}$. The impedance was below $5 \mathrm{~K} \Omega$.

3) Participants: Twenty five students (fifteen males, ten females) from university of International Islamic University Malaysia participated in the experiment. Before the experiment began they were asked to fill up provided informed consent form, all subjects had no personal history of neurological disorders. They had been given a monetary for their participation.

TABLE I

Time Frame Protocol EEG and ECG electrodes placements

\begin{tabular}{|c|c|c|c|c|c|c|c|c|c|c|c|}
\hline \multirow[b]{2}{*}{ 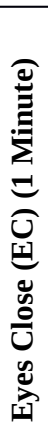 } & \multirow[b]{2}{*}{ 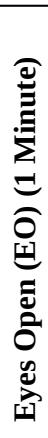 } & \multicolumn{4}{|c|}{ IAPS } & \multirow[b]{2}{*}{ 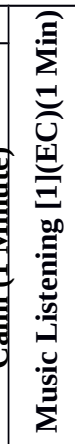 } & \multirow[b]{2}{*}{ 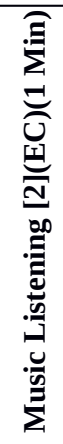 } & \multirow[b]{2}{*}{ 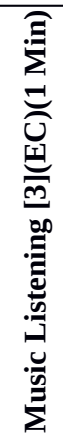 } & \multirow[b]{2}{*}{ 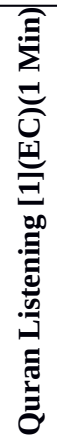 } & \multirow[b]{2}{*}{ 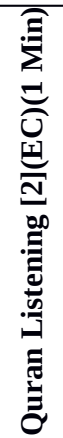 } & \multirow[b]{2}{*}{ 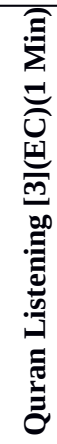 } \\
\hline & & 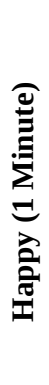 & 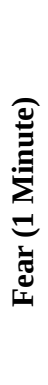 & 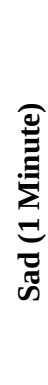 & 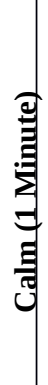 & & & & & & \\
\hline \multicolumn{2}{|c|}{2 Min } & \multicolumn{4}{|c|}{$4 \operatorname{Min}$} & \multicolumn{6}{|c|}{ Min / Total: 12 Minutes } \\
\hline
\end{tabular}

4) Procedures: Participants were instructed to set in a comfortable chair $80-100 \mathrm{~cm}$ away from laptop screen. They were asked to stay stable and minimize their movement as much as possible. The experiment began by eyes closed / eyes opened lasted one minute each. Then the subjects instructed to watch IAPS stimuli for four emotions (Happy, Fear, Sad and Calm) combined with the suitable music through headphone lasted one minute each. After the subjects watched emotion stimulus. 
Then they had been instructed to close their eyes to minimize eyes blinking artifacts and muscle movement while listening to the three excerpts of Quran recitation and to the three excerpts of a relaxing music through headphone lasted one minute each as shown in Table I.

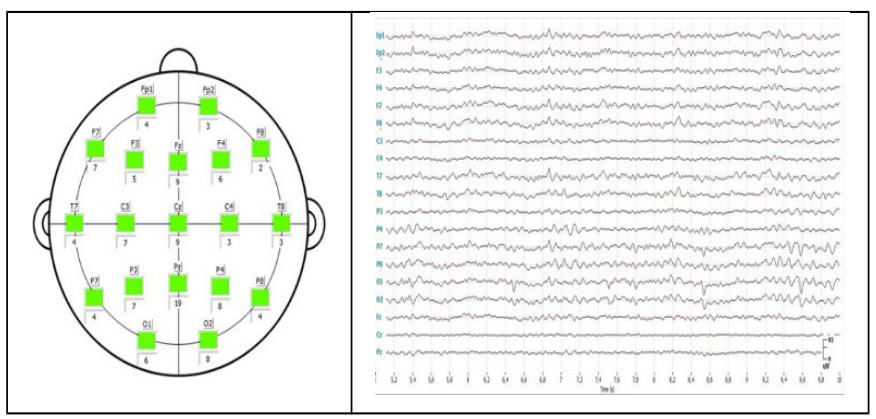

Fig. 2 BrainMarker software shows a good quality signals (green light) and the recorded signal is displayed live.

\section{B. Data Analysis}

Figure 3 shows the specific band for each wave and the condition that they appear on. As demonstrated in the flowchart in Figure 4, the analysis process started by importing the signal from the 19-channel EEG signal for all of the data, including emotions, Quran, music, eyes closed and eyes opened data. Then, we cut the frequency band for each wave, and after that, we apply the Butter filter in MATLAB for each band as in Figure 3, which represents channel F3 from the happy data for subject one. Finally, we take the average of the wave vector and plot it, focusing more on Alpha and Beta waves.

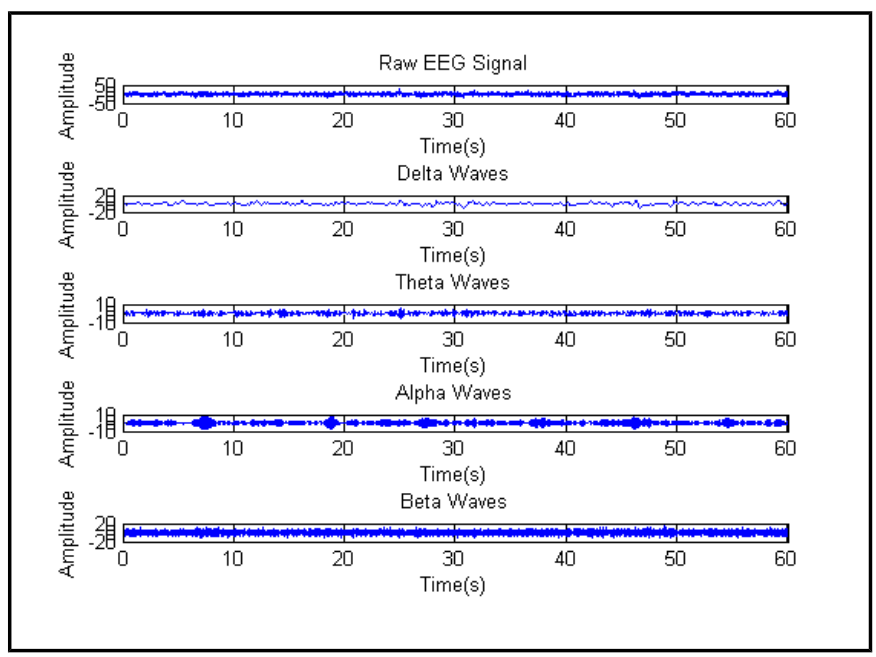

Fig. 3 an example of the brain waves analysis from subject 1 (happy -> channel F7)

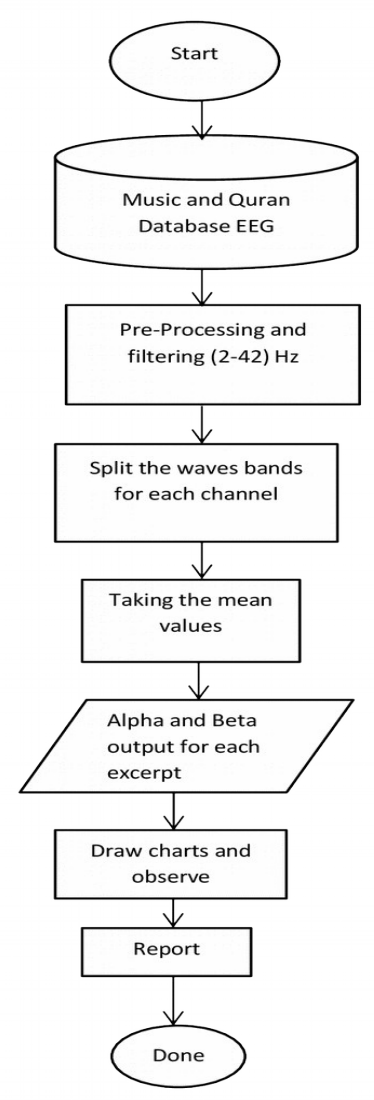

Fig. 3 a flowchart for the analysis procedures for brainwaves

\section{RESULT AND DISCUSSION}

As mentioned in the literature, increased alpha wave activity is definitely a desired outcome for researchers because alpha waves are essentially dominant when the mind is in a peaceful and relaxed emotional state or sometimes in a meditative state-bearing in mind that the subject is not tired or asleep. In contrast, beta waves are dominant when the mind is in a normal waking state involving awareness, when attention is directed towards cognitive tasks and outward activities.

Beta waves are a fast activity and are found when we are attentive, alert, involved in problem solving, making judgements or decisions, and engaged in concentrated mental activity. The performance of the alpha and beta waves of subjects' basic emotions is shown in Table II. To shed light on the calmness emotion, it is clear that $68 \%$ of the subjects produce a higher alpha magnitude than beta magnitude. The values of the four emotions were calculated, and the highest value is for the calm alpha band, which is highlighted with a light 
green colour in Table II. These data provide further support to the hypothesis that higher alpha wave magnitude is associated with calm and relaxed emotions. We further analysed alpha and beta brainwaves for the Quran verses and music tracks, and the results show that the alpha magnitude is higher than the beta magnitude when listening to the Quranic recitation in most subjects, as shown in Figure 5.

TABLE II

Alpha and Beta Brain Waves magnitude for four basic emotions

\begin{tabular}{|c|c|c|c|c|c|c|c|c|}
\hline \multirow{2}{*}{$\begin{array}{c}E \\
\alpha \& \\
\beta\end{array}$} & \multicolumn{2}{|c|}{ Happy } & \multicolumn{2}{|c|}{ Calm } & \multicolumn{2}{|c|}{ Fear } & \multicolumn{2}{|c|}{ Sad } \\
\hline & Alpha & Beta & Alpha & Beta & Alpha & Beta & Alpha & Beta \\
\hline s1 & 0.00033 & 0.0005 & 0.0003 & 0.0008 & 0.0002 & 0.0004 & 0.0003 & 0.0005 \\
\hline s2 & 0.00023 & 0.0004 & 0.0002 & 0.0003 & 0.0004 & 0.0002 & 0.0006 & 0.0013 \\
\hline s3 & 0.00080 & 0.0006 & 0.0009 & 0.0002 & 0.0003 & 0.0001 & 0.0006 & 0.0005 \\
\hline S4 & 0.00094 & 0.0010 & 0.0011 & 0.0003 & 0.0009 & 0.0005 & 0.0005 & 0.0005 \\
\hline s5 & 0.00058 & 0.0004 & 0.0007 & 0.0004 & 0.0005 & 0.0007 & 0.0005 & 0.0013 \\
\hline s6 & 0.00042 & 0.0002 & 0.0005 & 0.0003 & 0.0006 & 0.0006 & 0.0003 & 0.0010 \\
\hline s7 & 0.00032 & 0.0005 & 0.0002 & 0.0002 & 0.0002 & 0.0002 & 0.0005 & 0.0003 \\
\hline s8 & 0.00037 & 0.0003 & 0.0003 & 0.0004 & 0.0008 & 0.0002 & 0.0009 & 0.0002 \\
\hline s9 & 0.00037 & 0.0004 & 0.0005 & 0.0002 & 0.0002 & 0.0003 & 0.0008 & 0.0004 \\
\hline S10 & 0.00088 & 0.0005 & 0.0003 & 0.0002 & 0.0004 & 0.0005 & 0.0003 & 0.0004 \\
\hline S11 & 0.00098 & 0.0007 & 0.0018 & 0.0007 & 0.0009 & 0.0005 & 0.0015 & 0.0003 \\
\hline $\mathrm{S} 12$ & 0.00026 & 0.0005 & 0.0005 & 0.0004 & 0.0006 & 0.0016 & 0.0002 & 0.0007 \\
\hline $\mathrm{s} 13$ & 0.00017 & 0.0003 & 0.0006 & 0.0006 & 0.0004 & 0.0002 & 0.0015 & 0.0011 \\
\hline S14 & 0.00045 & 0.0003 & 0.0008 & 0.0009 & 0.0004 & 0.0005 & 0.0004 & 0.0003 \\
\hline S15 & 0.00059 & 0.0010 & 0.0008 & 0.0002 & 0.0009 & 0.0006 & 0.0011 & 0.0003 \\
\hline S16 & 0.00029 & 0.0005 & 0.0007 & 0.0009 & 0.0001 & 0.0003 & 0.0002 & 0.0003 \\
\hline S17 & 0.00069 & 0.0003 & 0.0003 & 0.0003 & 0.0002 & 0.0002 & 0.0006 & 0.0005 \\
\hline S18 & 0.00043 & 0.0006 & 0.0006 & 0.0009 & 0.0009 & 0.0005 & 0.0004 & 0.0005 \\
\hline S19 & 0.00025 & 0.0008 & 0.0003 & 0.0005 & 0.0002 & 0.0008 & 0.0007 & 0.0009 \\
\hline S20 & 0.00024 & 0.0005 & 0.0006 & 0.0005 & 0.0005 & 0.0007 & 0.0004 & 0.0004 \\
\hline S21 & 0.00037 & 0.0008 & 0.0013 & 0.0003 & 0.0003 & 0.0004 & 0.0002 & 0.0001 \\
\hline S22 & 0.00037 & 0.0006 & 0.0005 & 0.0004 & 0.0008 & 0.0009 & 0.0002 & 0.0007 \\
\hline S23 & 0.00187 & 0.0002 & 0.0002 & 0.0002 & 0.0002 & 0.0003 & 0.0003 & 0.0003 \\
\hline S24 & 0.00113 & 0.0012 & 0.0011 & 0.0009 & 0.0020 & 0.0007 & 0.0005 & 0.0013 \\
\hline $\mathbf{S} 25$ & 0.00020 & 0.0002 & 0.0002 & 0.0002 & 0.0002 & 0.0003 & 0.0001 & 0.0004 \\
\hline Avg & 0.00054 & 0.0005 & 0.0007 & 0.0005 & 0.0006 & 0.0005 & 0.0006 & 0.0006 \\
\hline
\end{tabular}

The alpha magnitude is almost as high as the beta magnitude for many of the subjects for tracks 1 and 2 . For many subjects, track 3 elicited a higher alpha magnitude than beta magnitude. However, there are exceptions, such as subjects $3,9,10$ and 12 , who generated higher alpha magnitudes than beta magnitudes for tracks 1 and 2 . Additionally, subjects $2,5,11,18$ and 21 generated higher alpha magnitudes than beta magnitudes for track 2 but not for track 1 , because of the space limitation so we have just chosen randomly four subjects.

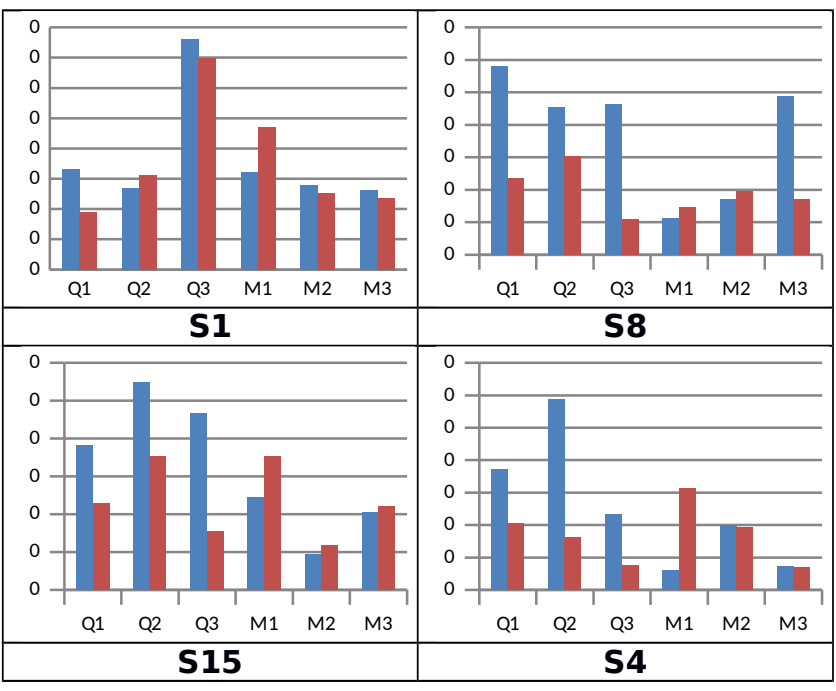

Fig. 5 Alpha (blue bars) and Beta (red bars) brainwave magnitudes (y-axis) for Quran verses (Q1, Q2 \& Q3) and music tracks (M1, M2 \& M3) for four selected subjects.
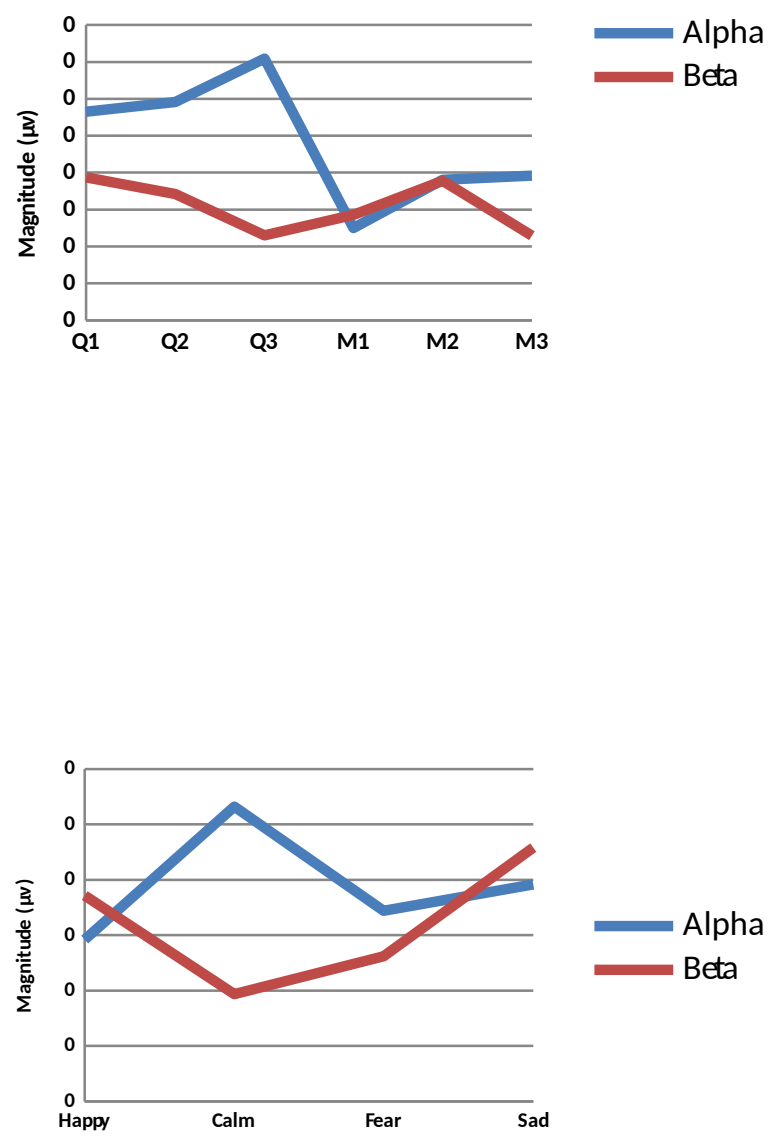

Fig. 6 Top: Alpha and Beta brainwaves magnitude for basic emotions for all subjects; Bottom: Alpha and Beta brainwaves magnitude for Quran verses and music tracks for all subjects 
The alpha and beta brainwaves for the Quran verses and music tracks were calculated. The results demonstrate that the alpha magnitude is higher than the beta magnitude when listening to the Quranic recitation, as shown in Figure 6. In contrast, the alpha and beta magnitudes are almost equal for music tracks 1 and 2. However, the third track showed a higher alpha magnitude than beta magnitude. Thus, Quranic recitation as sound therapy can improve brainwaves by generating high alpha activity, which is related to a resting and calm state. In contrast, the three selected types of best known relaxing music can also generate high alpha brainwaves but not as high as Quranic recitation.

\section{CONCLUSIONS}

Brainwave result analysis showed that Quran data generates higher alpha magnitudes then beta magnitudes, which reflects the calmness and relaxation of the subjects while listening to Quran recitation. Alternately, relaxing music data generated almost equal beta and alpha magnitudes but not as high as Quran recitation.

\section{ACKNOWLEDGMENT}

This project has been funded by Ministry of Higher Education Malaysia (MOHE) under Fundamental Research Grant Scheme (FRGS14127-0368).

\section{REFERENCES}

[1] Erkkilä, J., Punkanen, M., Fachner, J., Ala-Ruona, E., Pöntiö, I., Tervaniemi, M., ... Gold, C. (2011). Individual music therapy for depression: randomised controlled trial. The British Journal of Psychiatry: The Journal of Mental Science, 199(2), 132-9. http://doi.org/10.1192/bjp.bp.110.085431

[2] Kim, J., Wigram, T., \& Gold, C. (2008). The Effects of Improvisational Music Therapy on Joint Attention Behaviors in Autistic Children: A Randomized Controlled Study. Journal of Autism and Developmental Disorders, 38(9), 1758-1766. http://doi.org/10.1007/s10803-008-0566-6

[3] Lippi, D., Roberti di Sarsina, P., \& D'Elios, J. P. (2010). Music and medicine. Journal of Multidisciplinary Healthcare, 3, 137-41. http://doi.org/10.2147/JMDH.S11378

[4] Abdullah, A. A., \& Omar, Z. (2011). The Effect of Temporal EEG Signals While Listening to Quran Recitation.

[5] Yaacob, H., \& Wahab, A. (2012). EEG Affect Analysis based on KDE and MFCC, (June).

[6] Russell, J. a. (1980). A circumplex model of affect. Journal of Personality and Social Psychology, 39(6), 1161-1178. http://doi.org/10.1037/h0077714

[7] Zulkurnaini, N. A., Shilawani, R., Kadir, S. A., Murat, Z. $\mathrm{H}$. \& Isa, R. M. (2012). The Comparison between Listening to Al-Quran and Listening to Classical Music on the Brainwave Signal for the Alpha Band. http://doi.org/10.1109/ISMS.2012.60

[8] Lang, P. J., Bradley, M. M., \& Cuthbert, B. N. (2008). International affective picture system (IAPS): Affective ratings of pictures and instruction manual.Technical report $A-8$.

[9] Baumgartner, T., Esslen, M., \& Jäncke, L. (2006). From emotion perception to emotion experience: emotions evoked by pictures and classical music. International Journal of Psychophysiology: Official Journal of the International Organization of Psychophysiology, 60(1), 34-43. http://doi.org/ 10.1016/j.ijpsycho.2005.04.007

[10] Saari, P., \& Eerola, T. (2014). Semantic Computing of Moods Based on Tags in Social Media of Music, 26(10), 2548-2560.

[11] Smith, J. C., \& Ma, C. A. J. (2004). Mozart versus New Age Music: Relaxation States, Stress, and ABC Relaxation Theow, (3), 215-224.

[12] Yehuda, N. (2011). Music and Stress. Journal of Adult Development, 18(2), 85-94. http://doi.org/10.1007/s10804-010-9117-4 